\title{
The VIRGO PN population and the mass assembly in M87
}

\author{
Alessia Longobardi ${ }^{1,2}$, Magda Arnaboldi ${ }^{3}$ and Ortwin Gerhard ${ }^{2}$ \\ ${ }^{1}$ Kavli Institute for Astronomy and Astrophysics, Peking University, \\ 5 Yiheyuan Road, Haidian District, Beijing 100871, P. R. China, \\ email: alongobardi@pku.edu.cn \\ ${ }^{2}$ MPI für extraterrestrische Physik, Giessenbachstrasse 1, D-85741 Garching, Germany \\ email: alongobardi@mpe.mpg.de, gerhard@mpe.mpg.de \\ ${ }^{3}$ European Southern Observatory, Karl-Schwarzschild-Strasse 2, D-85748 Garching, Germany \\ email: marnabol@eso.org
}

\begin{abstract}
Cosmological simulations allow us to study in detail the evolution of galaxy halos in cluster environments, but the extremely low surface brightness of such components makes it difficult to gather observational constraints. Planetary nebulas (PNs) offer a unique tool to investigate these environments owing to their strong [OIII] emission line. We study the light and kinematics of the Virgo cluster and its central galaxy, M87, prime targets to address the topic of galaxy formation and evolution in dense environments. We make use of a deep and extended PN sample ( $\sim 300$ objects) that extends out to $150 \mathrm{kpc}$ in radius from M87's centre. We show that at all distance the galaxy halo overlaps with the Virgo intracluster light (ICL). Halo and ICL are dynamically distinct components with different parent stellar populations, consistent with the halo of M87 being redder and more metal rich than the ICL. The synergy between PN kinematic information and deep V/B-band photometry made it possible to unravel an ongoing accretion process in the outskirt of M87. This accretion event represents a non-negligible perturbation of the halo light, showing that this galaxy is still growing by accretion of smaller systems.
\end{abstract}

Keywords. galaxies: clusters: individual (Virgo cluster), galaxies: halos, galaxies: individual (M87), galaxies: kinematics and dynamics, planetary nebulae: general

\section{Introduction}

Galaxy halos are faint stellar components made of stars gravitationally bound to the individual galaxies. In galaxy clusters these halos may be surrounded by IC stars (Gonzales et al. 2005). The formation of ICL and extended halos around central cluster galaxies $(\mathrm{cD})$ is closely related to the transformation of galaxies in clusters (De Lucia et al. 2012) and, hence, their physical properties reflect the formation of the galaxies and their hosting clusters. When studying cDs we might wonder whether any photometrical and dynamical difference does exist between the central galaxy halo and the ICL. Usually no differentiation between them is possible and their physical properties are determined by treating them as a continuum. But is this really the case?

The Virgo cluster, the nearest large scale structure in the Universe, and its central galaxy M87 are prime targets to address the topic of galaxy/cluster formation and evolution, and to answer the question raised above. Virgo is a dynamically young cluster with a complex network of extended tidal features, suggesting that its core is not completely in equilibrium (Mihos et al.2005). At its centre lies the giant elliptical galaxy M87 with a $\mathrm{cD}$ halo that extends out to $\sim 150 \mathrm{kpc}$ in radius, and down to $27 \mathrm{mag} \operatorname{arcsec}^{-2}$ in the V-band. The observed blue colour gradients towards its outskirts (Rudick et al. 2010), hence the inferred metallicity and age gradients (Montes et al. 2014), are consistent with 
a late build up of its halo, suggesting that here we can find a great deal of information on the physical mechanisms governing the mass assembly in the Universe.

However, the most significant challenge to investigate galaxy halos far away from their centres is that these regions have a very low surface brightness, making it difficult for most of the traditional techniques to be successful. PNs, offer a unique tool to investigate these environments owing to their strong [OIII] emission line, whereby $\sim 15 \%$ of the luminosity of the central star is re-emitted in the forbidden [OIII] line at $\lambda 5007 \AA$ (Dopita et al.1992). Hence they make it possible to study M87 beyond its brightest regions, where the halo blends into the ICL.

\section{PN surveys in the Virgo core and M87}

Previous PNs studies in Virgo and M8\%. Several large-scale [OIII] $\lambda 5007 \AA$ surveys of intergalactic fields have been carried out with the goal of mapping the distribution, kinematics, and luminosity function of PNs in Virgo, starting from the discovery of ICPNs by Arnaboldi et al. (1996). The study of ICPNs constrained the fraction of the ICL to $10 \%$ in the Virgo core (Feldmeier et al.2003). This percentage drops in lower density regions suggesting that the ICL is poorly mixed and that Virgo is a dynamically young cluster (Castro et al. 2009, Aguerri et al. 2005). Moreover, using PN kinematics and spatial distribution, Doherty et al. (2009) gave evidence for the truncation of the luminous halo of M87 due to an earlier mass interaction, and they showed that PNs might be separable into components that are bound to either the potential of the galaxy or of that of the cluster.

The large Suprime-Cam@Subaru and FLAMES@VLT PN surveys. To further investigate these findings our group decided to carry out deep photometric and spectroscopic PN surveys, and gather a uniformly distributed PN sample containing $\sim 15$ times more data than any other PN sample in these regions. They are described in what follows.

We first carried out a photometric survey with Suprime-Cam@Subaru that covered a $0.43 \mathrm{deg}^{2}$ region centred on M87 (Longobardi et al.2013), obtaining images with a (on-band) narrow-band filter centred on the redshifted [OIII] $\lambda 5007 \AA$ emission line (at the Virgo cluster distance) and a (off-band) broadband V-filter. As consequence of their bright [OIII] and very faint continuum, extragalactic PNs can be identified based on their colour (on-off band mag) vs on-band mag properties. This led to a large and deep photometric sample of PNs, that we spectroscopically followed-up with the FLAMES@VLT. The 287 sources classified as PNs were identified from their narrow and symmetric redshifted $\lambda 5007 \AA[\mathrm{OIII}]$ emission line, the presence of the second $\lambda 4959 \AA$ [OIII] emission line, and the absence of significant continuum. Data were acquired with a spectral resolution of $\mathrm{R}=22500$. With this setup, the measured line-of-sight (LOS) velocities were obtained with an estimated median accuracy of $4.2 \mathrm{kms}^{-1}$.

\section{PNs as tracers of light and stellar populations}

The different dynamics of Virgo ICL and M87 halo. The study of the PN kinematics by Longobardi et al. (2015) (L15a) showed that the M87 halo and the Virgo ICL are dynamically distinct components with different velocity and density distributions. Using Gaussian fitting and robust sigma clipping procedures they showed that the line of sight velocity distribution (LOSVD) is bimodal and splits into two Gaussian components, one that feels the potential of the galaxy halo and one that feels the potential of the cluster.

The halo-ICL bimodality is shown in Fig. 1 where we plot the projected phase-space distribution for both halo (red asterisks) and IC (blue asterisks) PNs. We also show 


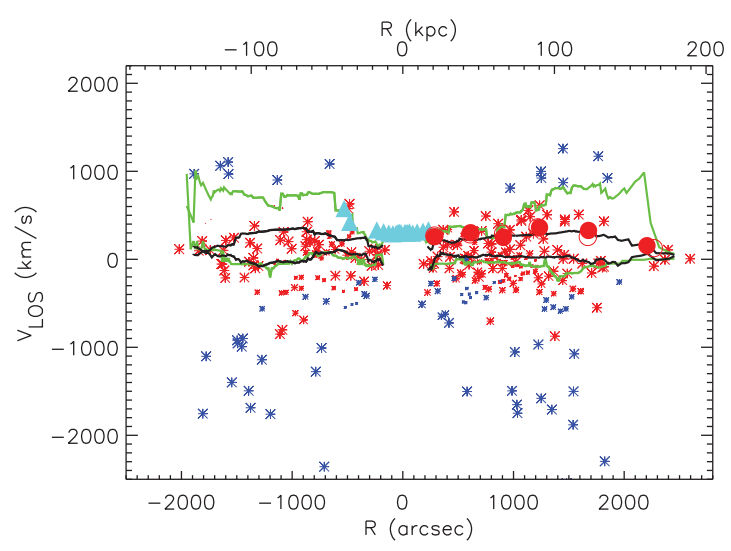

Figure 1. Velocity phase-space diagram $\mathrm{V}_{\mathrm{LOS}}$ vs. major axis distance R for both M87 halo (red asterisks) and IC component (blue asterisks) from L15a. Different point sizes represent different probability to belong to either the halo or the ICL. Black dashed-dot and black continuous lines depict the running average, and running dispersion computed from the 254 halo PNs weighted by their probability. Red full dots represent the robust estimate of the halo dispersion from L15, while red circle show the velocity dispersion when the 'crown' is statistically subtracted (see text). Green dashed-dot and green continuous lines represent the same quantities for the halo+IC component system. It is clear that including the ICL component leads to overestimate the velocity dispersion. Cyan triangles are IFU velocity dispersion data from Murphy et al. (2011, 2014) consistent with contamination from ICL (Longobardi et al. 2017).

the LOSVD 'running average', and 'running dispersion', i.e the first and second order moments of sub-sequences of 30 terms of the full data-set, for both the only halo (black lines) and the total halo+IC (green lines) system. While the overall mean velocity is not affected by the IC component, this population has an impact on the estimate of the dispersion. If halo and IC populations are not separated, the dispersion is overestimated, reaching, at its maximum, the Virgo sub-cluster A velocity dispersion $\sim 800 \mathrm{kms}^{-1}$.

The PN population of the Virgo ICL and M87 stellar halo. Virgo ICL and M87 galaxy halo not only do not share the same dynamics but they also differ in density profiles and underlying stellar populations. This is shown by the different properties of the PN population they are associated with, such as the $\alpha$-parameter, i.e. the number of PNs per bolometric luminosity, and the planetary nebula luminosity function (PNLF). The M87 halo is more centrally concentrated and it is characterised by a smaller $\alpha$-parameter than the ICL, typical of older and more massive systems. Moreover the ICL PNLF shows an overall shallower gradient than the halo PNLF and has a dip present at $\sim 1-1.5$ mag fainter than the bright cutoff. This is an evolutionary feature that is observed only in luminosity functions of younger and star forming systems. All these evidences lead to the conclusion that M87's halo and Virgo's ICL have two different parent stellar populations, consistent with the halo being redder and more metal rich than the ICL (L15a).

PNs as tracers of galaxy evolution: the 'crown' of M87 It is now known that the M87 halo shows kinematic signatures of accretion. Using PNs Longobardi et al. (2015) (L15b) showed that the M87 velocity phase-space is complex with a kinematic substructure that can be seen as cold features in a space dominated by random motion as consequence of a not complete phase-mixing of a disrupted satellite. In the light this substructure can be seen as a 'crown' shaped features, $70 \mathrm{kpc}$ north the M87's centre, causing an increase in light by $\gtrsim 60 \%$. The crown represents the densest region of the entire event that extends $\sim 50 \mathrm{kpc}$ along the major axis, where we also observe a variation in the colour: along the minor axis where no crown PNs are observed the galaxy is redder (Fig 2). 

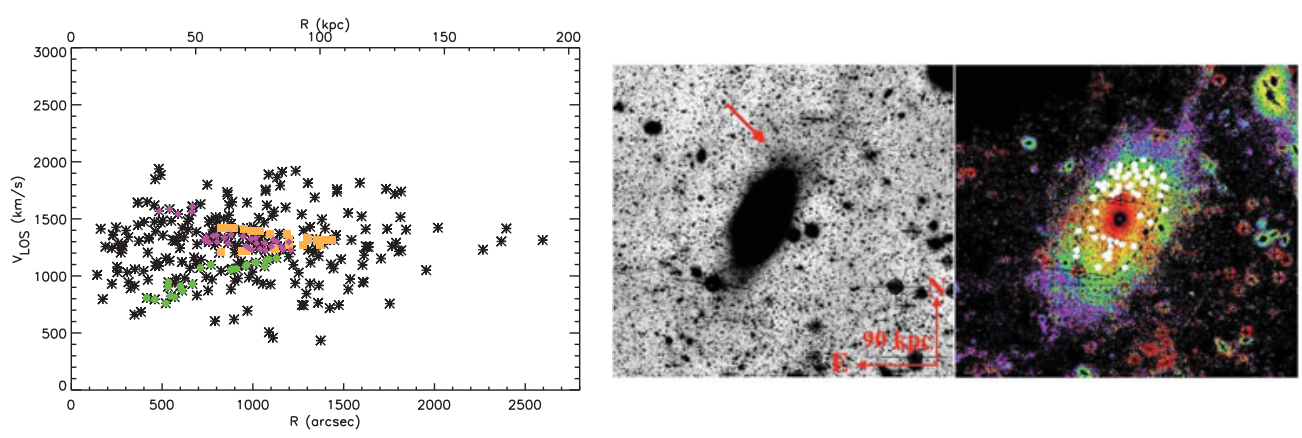

Figure 2. Left panel Velocity phase-space for the PNs (black asterisks) in the halo of M87. The green circles and magenta diamonds show the PNs associated with the crown substructure $(\mathrm{L}+15 \mathrm{~b})$. Orange squares show a GC substructure from Romanowsky et al. (2012). Central panel. Unsharped masked image of M87. The crown is visible at distance $60-90 \mathrm{kpc}$ along the major axis, NW of M87. Right panel: (B-V) colour image of M87 (Mihos et al. 2016) with substructure PNs overplotted (white dots). The ellipse indicates the isophote at $90 \mathrm{kpc}$. The crown is found in a region where $(\mathrm{B}-\mathrm{V}) \sim 0.8$, bluer than on the minor axis.

\section{Conclusions}

PNs represent a great tool to investigate regions in space characterised by very low surface brightnesses and where we can find signatures of the physical processes involved in the formation and evolution of astronomical objects. At the Virgo cluster core, PNs allowed us to trace the transition region between M87 galaxy halo and Virgo ICL, showing us that the M87 stellar halo is distinct from the surrounding ICL in its kinematics, density profile, and parent stellar population. PNs also traced an accretion event in M87 that has caused an important modification of its outer halo in the last Gyr. This accretion event, first revealed as kinematic substructure and then revealed as feature in the light, shows us that beyond $\sim 60 \mathrm{kpc}$ M87's halo is still assembling, and the PN kinematics reflects the dynamical complexity of a system still growing.

\section{References}

Aguerri, J. A. L., Gerhard, O. E., Arnaboldi, M., et al. , 2005 AJ, 129, 2585

Arnaboldi, M., Freeman, K. C., Mendez, R. H., et al. , 1996 ApJ, 472, 145

Castro-Rodríguez, N., Arnaboldi, M., \& Aguerri, J. A. L. , 2009 A $\& A, 507,621$

De Lucia, G., Weinmann, S., Poggianti, B. M., Aragon-Salamanca, et al. , 2012 MNRAS, 423, 1277

Doherty, M., Arnaboldi, M., Das, P., et al. , 2009 A $\xi A, 502,771$

Dopita, M. A., Jacoby, G. H., \& Vassiliadis, E. , 1992 ApJ, 389, 27

Feldmeier, J. J., Ciardullo, R., Jacoby, G. H., \& Durrell, P. R. , 2003 ApJS, 145, 65

Gonzalez, A. H., Zabludoff, A. I., \& Zaritsky, D. 2005, ApJ, 618, 195

Longobardi, A., Arnaboldi, M., Gerhard, O., et al. , 2013 A\&A A, 558, A42

Longobardi, A., Arnaboldi, M., Gerhard, O., \& Hanuschik, R. , 2015a A\&3A, 579A, 135L

Longobardi, A., Arnaboldi, M., Gerhard, O., \& Mihos, J., C. , 2015b A\&A, 579L, 3L

Longobardi, A., Arnaboldi, M., Gerhard, O. \& C. Pulsoni , 2017 A\&A, in prep.

Mihos, J. C., Harding, P., Feldmeier, J., \& Morrison, H. , 2005 ApJ, 631, L41

Mihos, J. C., Harding, P., Feldmeier, J., et al. , 2016 arXiv e-prints, 1611.04435

Montes, M., Trujillo, I., Prieto, M. A., \& Acosta-Pulido, J. A. , 2014 MNRAS, 439, 990

Murphy, J. D., Gebhardt, K., \& Adams, J. J. , 2011 ApJ, 729, 129

Murphy, J. D., Gebhardt, K., \& Cradit, M. , 2014 ApJ,785,143

Romanowsky, A. J., Strader, J., Brodie, J. P., et al. , 2012 ApJ, 748, 29 
Rudick, C. S., Mihos, J. C., \& Harding, P. , 2010 ApJ, 720, 569

\section{Discussion}

Q: Do globular clusters trace the same kinematics and substructures as PNe?

Q: The chevron that you show is like the tail of a comet. Is there any evidence for the remains of the shredded galaxy? Might a wavelet analysis help find theremnant? 